\title{
ENTREVISTA
}

\section{La franc-maçonnerie britannique et la notion de secret dans l'Angleterre du XXème siècle, De la seconde Guerre Mondiale aux années 2000}

La investigadora Amanda Brown-Peroy ha defendido una Tesis doctoral cuyo título es "La franc-maçonnerie britannique et la notion de secret dans l'Angleterre du XXème siècle, De la seconde Guerre Mondiale aux années 2000" en la Université Bordeaux Montaigne en Francia el 21 de octubre de 2016.

Amanda Brown-Peroyest née en 1986 à Royan, en France.

El autor nos ha concedido la siguiente entrevista.

Pouvez-vous nous indiquer les raisons pour lesquelles vous avez choisi d'entreprendre votre recherche sur la Franc-maçonnerie?

A chaque fois que j'évoque mon sujet de recherche, on me demande ce qui m'a poussée à m'intéresser à la franc-maçonnerie. Les réactions lorsqu'on évoque le thème du secret en franc-maçonnerie ne démentent pas l'affirmation selon laquelle tout le monde aime les complots. A vrai dire, je ne connaissais pas forcément bien la franc-maçonnerie, avant d'en faire mon sujet de mémoire de Master en 2011, mais j'étais passionnée par certains courants de pensée, ou organisations, qu'on peut associer de façon très lâche à la franc-maçonnerie, comme les Templiers par exemple. Au fil de mes études en langue, littérature et civilisation anglophone, j'ai été confrontée à plusieurs reprises à la maçonnerie dans différents contextes, notamment autour du débat sur l'abolition de l'esclavage (durant lequel sont intervenus les maçons, prenant d'ailleurs part des deux côtés du débat.) Ceci me laissa entrevoir que le fait maçonnique avait été au cœur des enjeux et débats de société, et éclairait certainement d'un jour particulier plusieurs épisodes de l'Histoire. C'est ainsi qu'en choisissant un sujet de mémoire de Master, je me suis naturellement tournée vers ce qu'on appelle de manière globale les sociétés secrètes. Au fur et à mesure que mon sujet se précisait, je me suis rendue compte que le problème majeur de ces sociétés dites secrètes était précisément qu'elles étaient trop privées, et leurs archives seraient par conséquent très difficilement consultables. Il serait alors compliqué de baser ce travail de recherche sur des sources primaires. C'est alors que la franc-maçonnerie se présenta comme une alternative idéale, puisque ses archives 
sont beaucoup plus accessibles. Cela fait maintenant plusieurs décennies que la francmaçonnerie constitue un objet d'étude, et compte-tenu de la pérennité de l'institution, les angles sont nombreux et les chercheurs peuvent l'aborder de différentes manières. Les débuts de la maçonnerie et le XVIIIe siècle notamment ont fait l'objet de nombreux travaux de recherche, et d'emblée je me suis aperçue que le XXe siècle, certainement du fait de l'aspect contemporain, était nettement moins représenté dans les travaux universitaires. J'ai donc pu rendre un travail novateur, sur un aspect de la maçonnerie qui était jusqu'alors largement inexploité.

Quelles sont les sources que vous avez utilisées?

Mon travail est bien sûr basé sur des sources primaires. Je me suis régulièrement rendu à Londres, à la bibliothèque de la franc-maçonnerie. Les documents et archives qui s'y trouvent sont très riches et j'ai pu les consulter librement. Par exemple, les archives individuelles de loges sont consultables facilement ainsi que les comptes-rendus des réunions trimestrielles de la Grande Loge Unie d'Angleterre, lors desquelles sont débattues les questions qui ont trait à la direction générale de l'institution. L'instance dirigeante, le Board of General Purposes, tranche sur les sujets qui lui sont soumis et donne l'impulsion à toutes les grandes loges provinciales sur la conduite à tenir. Ces comptes-rendus constituent donc un excellent point de départ pour ce qui est de connaître la position globale de l'institution. On peut rechercher par période les points d'intérêts dans ces ouvrages reliés, mais on ne peut pas faire l'économie de les lire en intégralité. J'ai également pu entretenir une correspondance avec plusieurs loges individuelles, ce qui m'a aidé à obtenir une vision plus détaillée. J'ai étudié ces sources primaires à la lumière des sources secondaires qui m'ont offert une vision d'ensemble du contexte historique et social, venant étayer des connaissances sur des sujets aussi variés que l'histoire sociale de la religiosité, la littérature de guerre, ou la sociologie du secret, inscrivant ainsi ce travail à la croisée de plusieurs domaines d'études, historique, politique, sociologique...

Quelles furent les principales difficultés auxquelles vous vous êtes confrontées? Comment les avez-vous surmontées?

En ce qui concerne les sources, il faut noter qu'un période de latence de 70 ans doit être respectée avant que les archives ne soient consultables par tous. Cette restriction m'a empêchée d'avoir accès à certains documents, notamment au niveau individuel de chaque loge, qui m'auraient été utiles.

Il est également délicat d'avoir accès à des informations factuelles, comme la composition des loges, notamment en termes politiques (un des aspects de ma thèse était de déterminer l'appartenance maçonnique au sein du parti conservateur). 
En ce qui concerne la rédaction pure, j'ai mis du temps avant de trouver un plan, une approche qui me permette de traiter tous les aspects que je voulais évoquer dans ma thèse : certains points se prêtaient mieux à être traités de manière chronologique, d'autres de manière thématique. J'ai finalement opté pour une approche chronologique, à l'intérieur de laquelle j'ai traité certains sujets de manière thématique (comme la place des femmes, l'appartenance politique, la relation à l'Eglise anglicane etc.)

Quelles sont les principales problématiques historiques que votre travail a résolues?

Les conclusions que j'ai pu tirer de ce travail sont multiples. J'ai tenté d'inscrire la francmaçonnerie dans le contexte politique et social de l'Angleterre du $\mathrm{XX}^{\mathrm{e}}$ siècle, puisqu'elle est selon moi indissociable de la société dans laquelle elle se trouve. En effet, la raison d'être de la franc-maçonnerie est de tendre à l'amélioration de la société. C'est pourquoi l'étude de la franc-maçonnerie éclaire la société britannique. Mais en se coupant volontairement d'elle, il semble que la maçonnerie anglaise se soit mise en situation d'échec. Le secret d'appartenance, le fait de taire l'appartenance maçonnique des frères, a pleinement joué le rôle qui était attendu d'une telle pratique pendant la période de guerre. Mais cette atmosphère de secret, de confidentialité qui a continué à entourer la franc-maçonnerie durant toute la seconde moitié du $\mathrm{XX}^{\mathrm{e}}$ siècle a finalement eu raison de ses spécificités. La maçonnerie anglaise, si scrupuleuse quant à ses landmarks, s'est finalement attirée les foudres de l'Eglise établie, elle qui avait toujours entretenu des liens forts avec elle, voit ses effectifs en perte de vitesse, et s'est pratiquement retrouvée en situation d'outrage au Parlement, un comble pour cette institution si traditionnellement associée à l'Establishment... Et tout cela, à cause d'une de ses valeurs, ironiquement celle qui a certainement sauvé la vie de nombreux maçons un demi-siècle auparavant : le secret. Lorsque la Grande Loge Unie d'Angleterre se rendit finalement compte que la fracture entre elle et la société était devenue béante, il était certainement trop tard. La perception négative était instaurée. Elle a tenté, et tente encore, de faire maintenant machine arrière : si le secret qui l'entoure est bien ce qui lui a porté préjudice, alors il faut à tout prix se départir de ce secret. C'est ce que font en ce moment même les conseillers en relations publiques et les dirigeants de la GLUA. Ils font tout leur possible pour dissiper ce qu'ils appellent les mythes qui entourent la franc-maçonnerie, mais est-ce vraiment une bonne chose ? La médiatisation et le désir de transparence dans les sociétés modernes font que les lignes bougent avec une rapidité phénoménale, et ce qui valait à la fin des années 1990 et le début des années 2000 ne vaut plus nécessairement aujourd'hui. Affirmer qu'il n'y a pas de poignée de main, pas de secret, et tout laisser voir au public n'est pas forcément dans l'intérêt des maçons britanniques. Le désir de découvrir ce qui est caché est un facteur puissant dans l'initiation, et tout en essayant de recruter de nouveaux membres, plus jeunes, la Grande Loge Unie d'Angleterre aurait peut-être à gagner, cette fois-ci, à conserver une part de secret... 
S'il vous plaît, pourriez-vous résumer l'essence de votre thèse en deux lignes?

Dans quelle mesure la vision que la société britannique porte sur la franc-maçonnerie a-t-elle influencé l'image que cette dernière donne à voir ? La notion de secret, au cœur des relations sociétales, a-t-elle pleinement joué le rôle qui lui était attribué avant la Seconde Guerre Mondiale, ou bien le secret a-t-il fini par desservir la maçonnerie ?

Quelles sont les leçons, à tous les niveaux, personnelles et professionnelles, que vous avez déduites de votre expérience de recherche?

La persévérance est sans doute la valeur la plus régulièrement mise à l'épreuve lors d'un travail de recherche de si long terme. C'est un travail solitaire, mais l'entourage a un rôle très important à jouer, de soutien et d'encouragement.

Et maintenant, quels sont vos projets professionnels?

Je suis titulaire d'un poste de professeur agrégé d'anglais à l'Université de Bordeaux depuis septembre 2018. Je continue, en parallèle, d'écrire des articles universitaires et grand public sur différents aspects de la franc-maçonnerie.

Esta entrevista se efectuó de forma telemática el 9 de diciembre de 2018.

Autores de la entrevista: Ricardo Martínez Esquivel e Yván Pozuelo Andrés, director y edit or de la REHMLAC+, doi: https://doi.org/10.15517/rehmlac.v10i2.35547 